\title{
Hemophilia in the 1990s: Principles of Management and Improved Access to Care
}

\author{
CAROL K. KASPER, M.D., PIER M. MANNUCCI, M.D., \\ VICTOR BULYZHENKOV, M.D., DOREEN B. BRETTLER, M.D., \\ AMPAIWAN CHUANSUMRIT, M.D., LILI HEIJNEN, M.D., \\ PARTTRAPOM ISARANKURA, M.D., PETER B.A. KERNOFF, M.D., \\ IAN PEAKE, M.D., KEVIN A. RICKARD, M.D., SAM SCHULMAN, M.D., \\ and CEES T. SMIT SIBINGA, M.D.
}

Physicians and scientists from several countries who are active in hemophilia care and in the World Federation of Hemophilia (WFH) conferred in March 1990 at the headquarters of the World Health Organization (WHO) to define current principles of hemophilia management and probe ways to improve access to such care in a wide array of countries. During the 1990s, one should expect to find excellent comprehensive hemophilia care programs in all industrialized countries. New or expanded programs should be promoted in countries with evolving health care systems.

From the Orthopaedic Hospital, University of Southern California, Los Angeles, California; A. Bianchi Bonomi Haemophilia and Thrombosis Centre and Institute of Internal Medicine, University of Milan, Italy; Hereditary Diseases Programme, Division of Noncommunicable Diseases and Health Technology, World Health Organisation; Medical Center of Central Massachusetts, Worcester, Massachusetts; Ramathibodi Hospital, Mahidol University, Bangkok, Thailand; Van Creveld Clinic, Bilthoven, and Rehabilitation Centre De Trappenberg, Huizen, The Netherlands; Royal Free Hospital and School of Medicine, London, United Kingdom; Department of Medicine and Pharmacology, University of Sheffield, Sheffield, United Kingdom; Roval Prince Alfred Hospital, Camperdown, NSW, Australia; Karolinska Hospital, Stockholm, Sweden; and Rode Kruis Bloedbank, Groningen-Drenthe, Groningen, The Netherlands.

Reprint requests: Dr. Kasper, Orthopaedic Hospital, 2400 South Flower Street, Los Angeles, California 90007.

\section{DESCRIPTION AND DIAGNOSIS OF THE DISEASE}

Hemophilia is a hereditary bleeding disorder affecting 15 to 20 of every 100,000 males born, with equal incidence in all ethnic groups and geographic areas that have been surveyed. Prevalence, which depends upon survival, varies according to available medical care. The disorder is inherited in a sex-linked recessive pattern. Thus, males are affected and females are carriers of the trait and occasionally affected, but usually mildly. Approximately one third of cases are caused by recent genetic mutations. Excessive, prolonged, or delayed bleeding occurs because of the absence, decrease, or deficient function of plasma coagulation Factor VIII or IX, causing hemophilia A (classic hemophilia, approximately $85 \%$ of cases) or hemophilia B (Christmas disease, approximately $15 \%$ of cases). The two types of hemophilia are clinically indistinguishable. In severe hemophilia, the most common site of bleeding is into the large joints of the limbs and the second most common site is into large muscles. With repeated bleeding, not controlled promptly by infusion of the deficient factor, progressive arthropathy and muscle atrophy ensue, leading to serious physical, psychologic, and social handicaps. ${ }^{1-4}$

Until recently, the main cause of death was hemorrhage, especially in the central nervous system. ${ }^{5-7}$ In countries with highly developed hemophilia care programs, therapy with plasma derivatives decreased mortality from bleeding. In the past decade, the foremost 
cause of death has been acquired immune deficiency syndrome (AIDS) due to infection through use of contaminated plasma products. ${ }^{8-11}$ Liver disease secondary to hepatitis also was a serious complication of treatment. Thus, improvement in survival and increasing average age were seen in hemophiliacs receiving plasma derivative therapy during the 1950s through 1970s, but a decrease in survival was seen in the latter half of the 1980s and is expected to continue into the 1990s because of deaths from AIDS. ${ }^{7}$ With introduction of viral-inactivated plasma derivatives in the mid-1980s, excellent life expectancy can be predicted for patients not previously infected, who include, of course, patients born in the 1980 s and coming of age in the early 21 st century. This cohort of patients, if treated by current standards, should reach adulthood in good physical condition, autonomous and fully active, and able to take similar positions in society to their nonhemophilic brothers.

The diagnosis of hemophilia is considered if a person with excessive bleeding has a family history of the disorder, or without such a history, if the bleeding pattern suggests the condition. In families with a history of hemophilia, infants should not be circumcised until the disorder has been excluded by specific laboratory tests. Females who might be carriers should have assays of the relevant clotting factor, because some carriers have clinically significant subnormal factor levels.

Severe hemophilia is usually clinically obvious and does not present a diagnostic dilemma. Persons with mild hemophilia may have prolonged bleeding only after notable trauma or surgical operations. Some persons are found to have mild hemophilia because a routine preoperative screening test, such as the activated partial thromboplastin time (APTT), is abnormal. Factor tests available at a specialized laboratory should include assays for Factors VIII, IX, and XI, and tests that distinguish and define von Willebrand's disease, such as the ristocetin cofactor test, ristocetin-induced platelet aggregation, and an immunologic test for von Willebrand factor antigen. To reduce variation in factor assays in specialized clinical coagulation laboratories, internal quality control protocols should be established and participation arranged in national and international quality assurance schemes. Reference plasmas can be calibrated against the International Standard for Factor VIII and von Willebrand Factor in Plasma.

Laboratories in expert hemophilia centers also can screen patient plasma for antibodies (inhibitors) to coagulation factors, which develop in some patients after treatment, and often are suspected when a patient does not respond adequately to plasma derivative treatment. The potency of the inhibitor can be measured using a standardized quantitative assay. As with factor assays, inhibitor measurements may vary from one laboratory to another because of differing reagents and variations in technique. Most commonly, the Bethesda system is used to quantitate inhibitors. ${ }^{12}$

\section{CURRENT MANAGEMENT OF HEMOPHILIA}

\section{Comprehensive Centers}

Optimum management of hemophilia is achieved through specialized expert interdisciplinary comprehensive care centers, which supervise local medical care and patient self-infusion of plasma derivatives. Centralized supervision gives patients with a rare disease access to knowledgeable experts in an efficient manner. Improved therapies can be instituted quickly.

Centers are usually located within general hospitals, although some successful ones have been established in other settings. A fundamental requirement is a designated physical area where hemophiliacs may expect a sympathetic and knowledgeable reception. Preferably, all staff are based in or near the center so that patients can receive all needed treatment at one place and on one occasion. Staff meet regularly to discuss and plan patient management. Care of acute problems such as bleeding episodes must be prompt, appropriate, and available at all hours.

Frequency and purpose of visits to a comprehensive center depend on patient circumstances. Newly diagnosed patients, often babies, are seen frequently. Routine care such as immunizations may provide an opportunity to educate parents about hemophilia. When a child is a few years old, families are often prepared to take over infusion of plasma derivatives. They are taught about signs and symptoms of bleeding, management of emergency situations, decisions about which hemorrhages require center treatment, preparation of the plasma derivative for infusion, calculation of dosage, safe venipuncture and infusion techniques, and the appropriate method of maintaining communication with the center about bleeding episodes. Such training may take many hours over several days or weeks. When a child reaches the age of about 10 years, he can often be taught to take over his own infusions. Adolescents and adults without complications may be seen at the center for routine reevaluation and advice yearly.

Scheduled center evaluations typically include interval history and physical examination with recommendations for plasma derivative use; evaluation of the musculoskeletal system with recommendations for fitness programs, therapeutic exercise, or orthotic devices; examination of the teeth with plans for appropriate care (because hemophiliacs often have avoided dental care, or have had difficulty finding a willing dentist, for fear of bleeding); and a conversation about problems of personal and family adjustment, school or work achievement, and 
(if necessary) finances of medical care. A nurse-coordinator often serves as the primary contact for patient and family, integrates care, and arranges all special consultations.

Patients with special problems, such as unstable musculoskeletal disability, poor psychologic or social adjustment, or human immunodeficiency virus (HIV) infection require more frequent visits. When care of HIVinfected patients is integrated into center activities, continuity of care is maintained for the patient. Consultation is obtained as needed from specialists in infectious disease or other fields. Additional funds are needed for care of these patients, including additional laboratory tests (such as counts of CD4 lymphocytes) used to monitor the stage of infection, and drugs, such as zidovudine and pentamidine, which slow the progress of HIV infection or prevent opportunistic infections.

\section{Hematological Management}

Hematologists have been involved intensively with the care of hemophiliacs for decades and protocols for hematologic management have been described in generally available sources. ${ }^{13}$ Some issues remain unresolved. One such issue is the minimum effective dose of Factor VIII (or Factor IX) for hemostasis. Doses of Factor VIII in the form of concentrate used in some industrialized nations in the 1970s and 1980s were generous. Through close observation at a boarding school for boys with hemophilia, ${ }^{14}$ dosage has been related to the probability of rapid hemostasis, depending on severity and duration of bleeding and on the susceptibility of any particular joint in a particular boy to frequent hemorrhage, thus suggesting that minimal adequate dosage must be judged for each hemorrhage in each patient. To conserve factor products, close communication between patient and physician or nurse is needed.

Another unresolved issue is the relationship between total dosage of clotting factor received and longterm musculoskeletal condition. Studies now in progress to define that relationship as well as the results of prophylactic infusions from an early age will help define the amount of musculoskeletal damage that can be prevented, and the price of prevention.

Of major importance is elimination of blood-borne infections, which has become possible through physical or chemical viral inactivation processes, such as heat or solvent detergent treatment applied to clotting factor concentrates. ${ }^{15,16}$ Furthermore, various chromatographic processes are being used to produce Factor VIII and IX concentrates of very high purity. ${ }^{17}$ Use of highly purified Factor IX concentrate in hemophilia B may avoid the excess thrombogenicity (for example, deep vein thrombosis) sometimes associated with concentrates containing other clotting factors in addition to Factor IX. Factor VIII concentrates prepared by recombinant DNA techniques have been developed recently and are in large-scale clinical trials. Efficacy has been demonstrated, but long-term safety studies continue. Advances in production technique will affect cost and may determine whether recombinant coagulation factors will replace treatment with plasma derivatives. ${ }^{18}$

Blood-borne infections also can be avoided in patients with mild hemophilia A or von Willebrand's disease by use of the synthetic vasopressin analogue, desmopressin (DDAVP), which stimulates release of Factor VIII and von Willebrand factor from storage sites. ${ }^{19}$ The drug is usually given by the intravenous route, but recently more convenient routes of administration, including subcutaneous injection and nasal sprays, are proving to be effective.

Inhibitor antibodies that arise against the deficient factor create major impediments in the control of bleeding. ${ }^{20}$ In a majority of patients, inhibitors can be suppressed with regimens of immune tolerance induction, which utilize regular administration of the factor concentrate, occasionally together with immunosuppressive drugs. Ways to control bleeding in patients with inhibitors are being studied intensively. Concentrates used include high doses of human Factor VIII, or porcine Factor VIII (because human inhibitors inactivate nonhuman Factor VIII to a much lesser extent than they inactivate human Factor VIII) and activated prothrombin complex concentrates (which may contain activated factors or complexes of factors that bypass the need for Factor VIII). There are currently under trial separate components of prothrombin complex that also bypass Factor VIII, notably Factor VIIa, now made by recombinant technology. Reduction of inhibitor levels by adsorption of antibodies from extracorporeal plasma during plasmapheresis also is available at some sites.

The future holds the hope of cure. A few patients undergoing liver transplantation subsequently have produced normal levels of the formerly deficient factor. The mortality rate of this procedure is substantial and patients must endure life-long immunosuppressive therapy. Thus, such a procedure is justifiable only for selected patients with end-stage liver disease. Although organ transplantation may not be feasible for the hemophilic population in general, gene transplantation may become possible. It is already possible to introduce the Factor VIII or Factor IX gene in human cell lines, which then synthesize functional coagulation factors. ${ }^{21,22}$ If the many technical problems of gene transplantation can be solved, such procedures may put an end to the need for coagulation factor replacement.

\section{Management of Musculoskeletal Problems}

Functional disorders caused by the sequelae of joint and muscle hemorrhages can be severe. The aim of good 
care is to reduce the incidence of early impairments, to limit or reverse progression into disability, and prevent the progression of disabilities into handicaps that impair the patient's ability to integrate fully into society. ${ }^{23} \mathrm{Com}$ prehensive care center staff should include persons dedicated to prevention and treatment of musculoskeletal disorders, who examine patients at least once a year, assessing range of motion of joints and strength of muscles, so that problems may be found at an early stage where prevention of impairments is still possible.

Patients with hemophilia should be advised to undertake regular physical activities and participate in sports to develop and maintain a strong musculature. Patients with strong muscles are less likely to sustain joint or muscle hemorrhages and, if such injury does occur, recover more rapidly. Parents of young patients must also be instructed about the benefits of exercise. Education is undertaken during visits to the center and reinforced by publications, with diagrams, easily understood by families and by local paramedical personnel. Sports advised for the patient are those with the least likelihood of trauma and the greatest potential for excercising all joints and muscles; examples of sports with minimal risk are badminton, fishing, rowing, table tennis, ballroom dancing, golf, swimming, and walking. Of these sports in which most hemophiliacs can participate safely, swimming is the most beneficial for the development of all muscles. Boys do not want to be different from their peers, so other sports also must be considered in which the physical, psychologic, and social benefits may outweigh the risk of trauma. These more risky sports should be supervised by experts who give the hemophiliac proper training, using reliable equipment. The patient also should be taught exercises to be done at home.

To prevent or reverse early impairment of function due to acute hemorrhage, the musculoskeletal specialist should use and teach such strategies as immobilization of the limb and use of ice during an acute hemorrhage, and, after hemostasis is secured, use of muscle setting exercises followed by active mobilizing exercises and active stretching.

For patients with disabilities such as unstable or painful joints or muscle wasting, specialized equipment such as splints and braces should be available. ${ }^{24}$ Stabilizing a joint by taping or similar inconspicuous means is more acceptable to youngsters than heavy braces. Useful modifications of shoes include heel lifts (when one leg is shorter than the other) or shock-absorbing soles (of great benefit for arthritis of the ankle). Wheelchairs and adaptations of bicycles and automobiles should be made, and adaptive furniture or architectural changes made at home or work.

When pain or contractures are causing disabilities, the orthopaedic surgeon should have facilities for opera- tive intervention. Hematologic care (laboratory facilities and plasma derivative therapy), operating rooms, postoperative facilities, and care should be of a high standard. A center and an orthopaedic surgeon familiar with the operative treatment of hemophilia patients are essential.

Several orthopaedic rehabilitative procedures have proven useful in hemophilia. When hemorrhages are recurrent and synovitis has developed that does not respond to conservative management (such as prophylactic factor replacement, anti-inflammatory medications, and a combination of protective splinting with isotonic exercises), radionucleotide synoviorthesis should be considered. When there is angular deformity at a painful deformed joint, osteotomy may be useful. A painful arthritic elbow with restricted range of motion may benefit from excision of an enlarged radial head. A painful, severely arthritic ankle joint with restricted range of motion is best treated with arthrodesis. Good range of motion in the hip and knee are important for the activities of daily living. Thus, if one of these joints is damaged by advanced arthritis, total joint replacement may be the treatment of choice.

Rehabilitation is not the work of the musculoskeletal specialist and hematologist alone. The social worker assesses the patient at the center and also visits the patient at home, contacts local authorities, school or work, and educates them about hemophilia to try to achieve integration of the person with hemophilia into society. Advice as to choice of school and vocation may be given in collaboration with a psychologist, who may want to test the patient. If there are disabilities that hinder the patient during his education or work, advice about possible adaptations, or other forms of schooling, or retraining, should be done as a joint effort among center staff members.

\section{GENETIC SERVICES}

\section{Genetic Counseling}

The aims of genetic counseling are to communicate, in a nondirective way, the features and recurrence risks of the disorder with information about possible alternatives, such as prenatal diagnosis and selective abortion. Emotional support for the person seeking information and the family must be provided during and after counseling regardless of the decisions that may have been made. In order to make decisions about reproduction, it is not sufficient to be armed with technical information alone. It is also necessary to deal with such emotions as guilt, resentment, anger, and fear.

Hemophilia centers provide the most appropriate setting for genetic counseling. Definitive counseling should be offered after puberty to girls who may be 
carriers. They should be invited to bring prospective partners in parenthood to counseling sessions so that decisions about reproduction are made together, avoiding subsequent guilt, blame, and resentment wherever possible. Women who request investigation and counseling for the first time when they are already married should be urged to attend with their husbands for the same reasons.

For some hemophiliacs and their relatives, abortion and attempted prevention of transmission of the defective gene are unacceptable on moral grounds. Some carriers regard selective abortion as implied rejection of a muchloved relative, such as a hemophilic father, brother, or son.

Carriers are faced with difficult decisions. Families have differing priorities and choose different courses of action. Whether a carrier has decided to have selective abortion or has opted to take the chance of having a hemophilic son, she should not be made to feel guilty about that choice by staff members who might have chosen differently for themselves.

\section{Carrier Detection and Prenatal Diagnosis}

Accurate carrier detection and early prenatal diagnosis are effective forms of control of an inherited disease. Obligate or proven carriers (daughters of hemophiliacs, mothers of hemophiliacs with antecedent hemophilic relatives) can be identified easily from the family pedigree. Among potential carriers are those women with a maternal hemophilic relative and those women (about $30 \%$ of mothers of hemophiliacs) with one hemophilic son and no further family history. Most of the latter have carried the trait unwittingly, due to a recent mutation in germ cells of their parents or grandparents, but some may have produced a unique ovum with the mutation. Some women may have somatic or germline mosaicism, complicating attempts at accurate diagnosis.

Phenotypic diagnosis of the carrier state is based on several observations. ${ }^{25,26}$ Obligate carriers of hemophilia have, on average, $50 \%$ of the normal plasma level of Factors VIII or IX measured biologically, that is, half of carriers have factor levels below the normal range. Because of the large normal range of Factors VIII and IX in plasma and the process of lyonization (random inactivation of one of the $\mathrm{X}$ chromosomes in females), a significant overlap between plasma factor levels found in normal women and obligate carriers is found. In hemophilia A, the evaluation can be improved by also measuring the plasma level of von Willebrand factor ( $\mathrm{vWF}$ ), which serves as a carrier of Factor VIII in plasma and also protects unactivated Factor VIII from proteolytic degradation. Thus, although the ratio of factor VIII to vWF is 1.0 in normal plasma, in carriers of hemophilia $\mathrm{A}$ it is close to 0.5 . Determination of this ratio is the best pheno- typic discriminant for carrier detection. Even so, some 5 to $10 \%$ of obligate carriers of hemophilia A still appear to be phenotypically normal given the best estimates of plasma Factor VIII and vWF levels.

Phenotypic prenatal diagnosis by measurement of Factors VIII or IX levels in fetal blood obtained at fetoscopy at 18 to 20 weeks' gestation has been very effective in skilled hands. ${ }^{27}$ However, since sampling is only possible in the second trimester, any resulting termination of an affected fetus is at a gestational age that many find unacceptable.

The ideal method for carrier detection and prenatal diagnosis is identification of the affected gene in possible carriers and in fetuses at risk, that is, genotypic analysis, which is rapidly supplanting phenotypic analysis wherever possible. ${ }^{28-30}$ Identification and tracking of the defective gene can be performed in two ways, either using restriction fragment length polymorphisms (RFLPs) or detection of specific DNA abnormalities. With RFLPs, normal polymorphic DNA variations within or close to the gene serve as markers for that gene. The usefulness of an RFLP depends on several factors, including frequency of polymorphism (heterozygosity) within a specific population. At many gene sites where heterozygosity is common in women of European descent, homozygosity is usual in women of Asian descent.

In hemophilia A, five intragenic RFLPs, all diallelic, have been described. Linkage disequilibrium (the tendency for one allele of one RFLP to associate with a particular allele of a second RFLP) results in an overall usefulness of these intragenic RFLPs in 70 to $80 \%$ of persons of European descent, and a lesser percentage of other racial groups. In addition, two linked polymorphic loci close to the Factor VIII gene locus have been observed. A crossover rate of about $5 \%$ between these loci, which are close together, and the Factor VIII gene limits their usefulness.

In hemophilia B, six intragenic Factor IX RFLPs and one useful linked RFLP have been described. Overall, by combined use of the intragenic RFLPs, some $90 \%$ of females of European descent are heterozygous. This figure is considerably lower in other racial groups.

Genomic diagnosis also can be accomplished by detection of the specific defect at the DNA level that causes hemophilia in a particular individual. Such detection is difficult in hemophilia A because of the size and complexity of the Factor VIII gene. Specific mutations causing hemophilia A include total and partial deletions, insertions and point mutations, detected in the factor VIII gene in about $10 \%$ of patients studied. ${ }^{31-33}$

For hemophilia $\mathrm{B},{ }^{34,35}$ the ability to detect DNA defects has improved dramatically in the past 2 years with the introduction of the polymerase chain reaction (PCR) technique of DNA amplification. With PCR, complete 
DNA sequencing of the eight exons within the Factor IX gene has allowed the defect to be found in almost all patients examined. As with hemophilia A, a series of deletions, insertions, and, in particular, point mutations have been described.

Genotypic analysis has a considerable advantage over phenotypic analysis, since it generally gives an absolute diagnosis when informative. Carrier detection by intragenic RFLP analysis can be considered to have a very high level of accuracy as, of course, has direct defect detection. Single tests are generally required and modern PCR technology means that only very small samples of blood or other tissue are required. Importantly, prenatal diagnosis can be performed in the first trimester, between 10 and 12 weeks gestation, by analysis of DNA obtained from chorionic villi samples. RFLP analysis, because it does not detect the gene defect but only a polymorphic marker close to it, does have certain limitations. Essential family members must be available to allow for unambiguous tracking, and certain females within the family must be heterozygous for at least one of the RFLPs available. Mistaken paternity can also occasionally cause errors of interpretation. RFLP analysis is of limited use in families with sporadic hemophilia (that is, no prior history of hemophilia) where it can diagnose the noncarrier state in some females but cannot rule out the carrier state in others. The advantages of RFLP analysis are that it is applicable to all types of hemophilia irrespective of the gene defect, and, with PCR technology, it is becoming a relatively simple, inexpensive, and quick procedure. In contrast to direct defect detection, RFLP analysis may be informative when none of the hemophiliacs in the kindred are alive or available.

DNA is extremely stable and can be stored for many years. Because genotypic analysis by direct defect detection invariably requires DNA from an affected hemophiliac, it is very important to obtain and store suitable samples, particularly in view of the current high mortality rate from AIDS in hemophiliacs in some countries.

\section{HEMOPHILIA PROGRAM DEVELOPMENT}

\section{General Observations}

The development of a hemophilia program requires, firstly, recognition of need and then the knowledge and enthusiasm necessary to secure and develop resources. Evidence of need is gathered by creating a register of patients with hemophilia and related coagulation disorders, identifying their demography, severity of illness, and unmet needs. Hemophilia care is an impossible financial burden for the affected individual and his family. Thus, public funding is needed. In applying for such funding, it may be helpful to point out that appropriate hemophilia care has been proved to prevent handicaps. In terms of outcome, therefore, the treatment of hemophilia may be considered a highly effective use of resources. In considering costs, it is important to assess the costs of nontreatment or undertreatment, such as dependency of the patient and his parents, which result in long-term economic burdens falling directly or indirectly on governments. In addition, plans for hemophilia care can be integrated with plans for improvement of transfusion and laboratory services and for hematologic services for other disorders, thus benefiting a much wider group of patients and enlisting the support of more health professionals.

Funding of a hemophilia program can be met in different ways. In countries with national health systems, hemophilia care is assumed to be covered. Supplementary funds, however, may be needed for specialized hemophilia centers. Systems requiring patient co-payment for medications and medical care must exempt persons with expensive lifelong disorders such as hemophilia. In some countries, intravenous infusion of blood products at home is forbidden by law; thus, legislation is needed to exempt hemophiliacs. In countries without national health systems, only employed persons have health insurance, thus excluding most hemophiliacs. In these countries, governments may be asked to list hemophilia among diseases causing exceptional social and financial burdens, thereby entitling patients to free medical care.

Hemophilia program development requires determined leaders. Typically, the initiator or developer of a program is a specialist physician with a main commitment within the fields of hematology or blood banking. Lay organizations (such as hemophilia societies, often led by parents of affected children and by adult patients) sometimes are the driving forces. In either instance, successful programs are difficult to establish and maintain without financial and other support services of governments.

Delivery of comprehensive care requires active collaboration of a variety of health care professionals. Ways must be found of involving such persons in hemophiliarelated problems, persuading them to make commitments of time and effort, and educating them in the principles of hemophilia management. The motivation for such staff is rarely financial. They are more often attracted by the intrinsic interest of the work, the potential for recognition of their expertise and contribution to a field of medicine in which relatively few people can be regarded as experts, and opportunities for academic studies.

The concept of the "hemophilia center" is now wellestablished as essential, but the specifications and functions of centers may differ greatly according to local circumstances. In countries with evolving health care systems, hemophilia centers are highly likely to be part of 
larger hematology services or be closely associated with blood banks. As programs mature, the organizational framework may shift toward fewer but larger centers with high levels of expertise and technology, but with less involvement in day-to-day aspects of primary care. Major causes of this shift in emphasis are improvement in education of the patient, the family, and local health practitioners, and expansion of supervised self-infusion programs in which family members or local medical personnel administer plasma derivatives quickly at the onset of hemorrhages. Self-treatment programs have the advantage of providing rapid hemostasis and thus minimal damage to tissues, minimal sequelae, and little disruption of normal schedules and activities. On the other hand, such programs shift control and responsibility away from the physician toward the patient and family. The former may be unhappy to lose control and the latter may not be prepared to accept responsibility.

The mainstay of hemophilia care is adequate availability of lyophilized virus-inactivated concentrates of plasma clotting factors. Cryoprecipitate and plasma, which are not viral-inactivated, sometimes are still used but are not ideal. Concentrate availability entails an adequate supply of plasma (obtained through separation of blood components or through plasmapheresis) and the necessary technology to fractionate it. Some countries develop and use their own fractionation facilities, either private or state-owned. Other countries contract with external fractionation facilities, which may be a good means to avoid unnecessary duplication of expense and effort. The goal is to provide enough concentrate to prevent most handicaps in all the hemophiliacs in the country.

\section{Role of Transfusion Services}

Countries with inadequate transfusion services are not ready for initiation of modern hemophilia care. Such care can be developed only where there is a well-organized and efficient blood transfusion network continuously providing adequate, safe blood products on an equitable basis. Cryoprecipitate and fresh-frozen plasma, the simplest blood products used to treat hemophilia, can be prepared easily as by-products from whole blood donations with elementary technology within the grasp of all countries. To prepare viral-inactivated products, the introduction of freeze-drying technology and heat treatment is important. The League of Red Cross and Red Crescent Societies (LRCS), WHO, and the International Society of Blood Transfusion (ISBT) stand ready to assist developing countries to shape their blood transfusion capabilities and establish an integrated quality assurance program. Problems in providing adequate transfusion services have increased because of the AIDS epidemic, since an additional financial burden is entailed to test for HIV. An international group, the Global Blood Safety Initiative (GBSI), initiated by WHO, LRCS, and ISBT, with input from WFH, has published its first recommendations on the structuring of safe and effective blood transfusion organizations, on the equipping and supply of laboratories and blood banks, and optimal use of essential blood components, plasma derivatives, and substitutes.

Self-sufficiency is the goal of an increasing number of national transfusion services. Human blood for transfusion should be regarded as a national resource to be shared by all on an equitable basis. The community is the basic shareholder and the medical profession is the principal protector of the resource. All appropriate segments of the population should be motivated to donate blood voluntarily. National health authorities should accept their fundamental responsibility to protect the blood donor and the patient by instituting good standards of practice and a mechanism for inspection, control, and registration.

A national fractionation policy is needed to facilitate production of the needed amount of quality plasma products, such as purified and safe Factor VIII and IX preparations, albumin, and gamma globulin, whether processed within the country or at another reliable location. One or more national reference laboratories should be organized and maintained for development of blood bank technology and for training and ongoing education of all blood bank personnel.

\section{Specific Proposals for Pilot Programs for Hemophilia Care}

Pilot programs for the establishment or betterment of hemophilia care have been a major interest of WFH. Such programs are small in scale and, of course, may not be successful in every instance. A country targeted for such a program should meet certain prerequisites, including the presence of a functioning blood transfusion system that has the potential for, or includes, some blood component production, and the presence of a key person who has espoused interest and willingness to provide hemophilia care. Pilot programs may be applied at two levels, at a primary level in a country that is about to initiate hemophilia care or at a secondary level in a country with an existing hemophilia program that wants to increase its degree of sophistication in hemophilia care. If such countries are also strategically placed in a particular geographic area, they might then serve as a reference center for that area and undertake the task of developing and improving hemophilia care in neighboring lands.

At the primary level, consideration should be given to Zimbabwe, where a transfusion service is present in 
Harare and a key person has been identified. Blood transfusion services and plasma fractionation facilities are found in many sites in the People's Republic of China, a highly qualified country and a high priority target for primary hemophilia care development.

A transfusion service exists in Indonesia and an interested physician has been identified, and in Vietnam active steps are being taken to rehabilitate their medical services and the Institute of Haematology in Hanoi has a strong interest in hemophilia care.

In the subcontinent of India, transfusion services exist in several cities and WFH has recently staged workshops in Calcutta, Trivandrum, Delhi, and Vellore. Progress in establishing hemophilia care, however, is slow.

In the Philippines, a transfusion service and considerable medical expertise exist, but hemophilia care is lacking in many parts of the country.

Attention to eastern Europe may be appropriate. Political barriers have fallen and in this atmosphere much can be done for hemophilia care in such countries as Poland, Romania, Hungary, Czechoslovakia, and Soviet countries.

In South America, WFH workshops recently have been staged in Bogota and Medellin in Colombia and one soon will take place in Santiago, Chile.

At the secondary level, several opportunities exist. In the Malay peninsula, transfusion programs exist in Kuala Lumpur, Malaysia, and also in Singapore, but the outreach to hemophilia care is limited. An excellent transfusion service exists in Bangkok with considerable modern technology and sophistication, thanks, in part, to the France's Cultural and Scientific Exchange Programme. Hemophilia care is good in Thailand, but this country now has a vast potential for expansion of hemophilia care as well as the potential ability to provide help to neighboring lands such as Vietnam, Indonesia, the Philippines, and perhaps even Sri Lanka, Pakistan, and India.

Costa Rica is an exemplary model of development of hemophilia care at the primary level and one of the triumphs of WFH. This country now wants to develop more sophisticated diagnostic methods, including carrier detection and prenatal diagnosis by genomic analysis, and also wants to develop new therapeutic products. If assisted to achieve these goals, Costa Rica can then help expand hemophilia care in Central America, northern South America and the Caribbean. Brazil (Rio de Janeiro) and Argentina (Buenos Aires) have standards of hemophilia care similar to those of Costa Rica and also have the potential to help expand hemophilia care in neighboring lands such as Uruguay, Paraguay, Bolivia, Peru, and other parts of Brazil and Argentina.
The International Haemophilia Training Centers (IHTCS) Committee of WFH is concerned with introducing and expanding the knowledge of medical care of people with hemophilia in the developing world. ${ }^{36} \mathrm{At}$ tempts to achieve this objective are made by two methods. The first involves the award of Fellowships to medical or paramedical persons with a proven interest in hemophilia in their own countries who are assured of being able to continue working in hemophilia care on return home after a Training Fellowship in a center of excellence. The second method is the utilization of workshops, in which a short but intense teaching exercise is conducted in a third-world country by persons selected by the Training Centre Committee. These workshops may consist of open consultative clinics, "wet" laboratory teaching, and lectures by the visiting team.

The IHTCs are a group of 23 strategically located hemophilia centers throughout the world. Each of these centers has comprehensive facilities for the treatment of persons with hemophilia and operates under the leadership of a Director who is a member of the IHTC Committee. (For geographic and linguistic reasons, a few centers were included that provide excellent primary care but may not have the advanced technology of the most industrialized countries; thus, they may qualify for secondary development). Most of these centers are located in university affiliated teaching hospital environments. Centers may be called on to accept Fellows for short training periods or to staff relevant workshops. Since its inception in 1970, over 80 Fellows have been trained in IHTCs.

In WFH/IHTC Workshops, formal instruction is not of itself sufficient to improve hemophilia care. WFH executive members consult with local or national health department officials to elicit a commitment to develop hemophilia care. Improvement of blood transfusion services and instruction on blood transfusion principles and organization is an essential part of workshop programs. To date, more than 22 workshops have been held under WFH auspices.

It may be advisable to develop combined WFHWHO workshops in two or three specific countries over the next 2 or 3 years, followed by establishment of early hemophilia programs in these countries. Subsequent objective follow-up assessments of achievements in these countries will guide implementation of similar proposals in other countries.

At the same time, it may be advantageous to mount a WFH-WHO effort to attain a more sophisticated level of development in two countries with already established hemophilia care programs. Such developments might concentrate on the control of hemophilia at the genetic level by emphasis on carrier detection and counseling. 
Other areas of concern might include improved diagnostic methods and improved management of musculoskeletal complications of hemophilia. Countries receiving such training should be encouraged to support and instruct the efforts of neighboring lands toward hemophilia care. The development of teaching, ambassadorial, and diplomatic skills would be involved.

\section{Example and Advice of Thailand}

Developing countries contain three-quarters of the world's population. They differ markedly in many ways, and implementation of hemophilia programs should be adjusted to suit each country and region. Thailand has been exceptionally successful in establishing an excellent hemophilia care network, beginning in 1978 to 1980 with conferences on bleeding disorders to interest and motivate health authorities.

Although the prevalence of hemophilia is low, patients bleed frequently and develop many problems with social implications. However, programs for care will gain support more easily if they are not limited to hemophiliacs but cover all bleeding disorders, such as those caused by infections, liver disease, malignancies, snake bites, and surgical and obstetric complications. Plans for hemophilia care should include improvement of blood banks, blood products, hematology and coagulation laboratories, and other medical facilities, thus benefiting many patients, raising the standards of medical care in general, and arousing the interest of a wide spectrum of health care providers. Hemophilia programs will be successfully implemented in the health system when government authorities recognize the significance of effective management of bleeding disorders and include them in their national health development plans and national health care system. A national center for blood diseases is recommended for supporting a hemophilia program. Linking it to the Red Cross, hematology society, hemophilia society, public and private granting agencies, and other institutes and organizations will strengthen its support.

A national hemophilia registry should be established as a basis for planning national health policy for the management of hemophilia. Such a registry should include not only the numbers and locations of hemophiliacs, but also a tally of disabled or handicapped persons and should track blood-transmitted infections such as HIV.

In developing countries, transportation of hemophiliacs to major hemophilia centers is very difficult, and health care resources including manpower and money are limited. Thus, promotion of home care is essential. Treatment can be provided with blood products pre- pared in the country, such as lyophilized cryoprecipitate or plasma, stored in home or village communal refrigerators, which also may be constructed within the country at less expense than with importation. Village health personnel can assume the responsibility of home care therapy by storing the blood product and performing the infusion. Home care in developing countries has reduced hospital admissions very markedly and, because care is given promptly, has reduced the severity and sequelae of hemorrhages and the total consumption of plasma products. Another benefit is the patient's psychologic independence, well-being, and improved quality of life.

The smallest local hospitals can provide care for simple problems. Larger regional hospitals can provide secondary medical care for such problems as major hemorrhages, dental extractions, and minor surgery, whereas a tertiary-care facility might house the hemophilia comprehensive care center and be responsible for such complicated problems as major surgery and management of patients with inhibitors. Management of hemophiliacs with HIV infection should be undertaken by the hemophilia care system in collaboration with the national AIDS program. Patient education and genetic counseling can be provided at every health care level described. Prevention of the disease is aided by carrier detection, which should be available in at least one center in each country. In populous countries, prenatal diagnosis of hemophilia should be made available, whereas smaller countries may use the services of a neighboring nation.

Improvement of blood banking and provision of an adequate supply of HIV-safe plasma products are vital to betterment of hemophilia care in developing countries. Collection of blood in plastic bags facilitates its separation into components, including plasma and cryoprecipitate, that can be lyophilized and supplied to local and regional hospitals. Plastic bags, however, usually are imported and expensive. The manufacture of such bags in developing countries is a desirable goal, but mastery of the technology is sometimes difficult. Indeed, each country or region should be encouraged to produce or manufacture its own equipment, reagents and therapeutic materials to the greatest extent possible in order to control costs. In large countries, plasma fractionation facilities can be developed locally. Smaller neighboring countries can share such facilities using their own raw plasma. Such a strategy would lower the cost of plasma products dramatically.

Ongoing training of medical personnel is important, and attention should be paid to career development of young staff members who will carry on the work initiated by their more senior colleagues. The assistance of WFH in providing training is very useful. The knowledge 
gained can be passed on to other colleagues in the same country and in neighboring countries.

\section{CONCLUSION}

Improvement of hemophilia care around the world by the end of the century depends on persistence in and support for proven methods of hemophilia care, on continued research into possible means of cure (gene therapy), and on expansion of hemophilia care to areas of the world where it is inadequate. In the latter pursuit, intense efforts expended by WFH on a few appropriate and receptive countries are deemed likely to have the most benefit.

\section{REFERENCES}

1. Bloom AL: Inherited disorders of blood coagulation. In: Bloom AL, DP Thomas (Eds): Haemostasis and Thrombosis. Churchill Livingstone, Edinburgh, 1987, pp 393-436.

2. Levine, PH: The clinical manifestations and therapy of hemophilias A and B. In: Colman RW, J Hirsh, VJ Marder, EW Salzman (Eds): Hemostasis and Thrombosis: Basic Principles and Clinical Practice. Lippincott, Philadelphia, 1982, pp 75-80.

3. Ljung R, P Petrini, IM Nilsson: Diagnostic symptoms of severe and moderate haemophilia A and B. Acta Pediatr Scand 79:196200, 1990.

4. Larsson A, IM Nilsson, M Blombäck: Current status of Swedish hemophiliacs: A demographic study. Acta Med Scand 212:195$200,1982$.

5. Aronson D: Cause of death in hemophilia A patients in the United States from 1968 to 1979. Am J Hematol 27:7-12, 1988.

6. Rosendaal FR, I Varekamp, C Smit, AHJT Brocker-Vriends, H van Dijck, JP Vandenbroucke, J Hermans, TPB Suurmeijer, E Briet: Mortality and causes of death in Dutch haemophiliacs, 197386. Br J Haematol 71:71-76, 1989.

7. Rosendaal FR, E Briet: The increasing prevalence of haemophilia. Thromb Haemost 63:145, 1990.

8. AIDS Group of the United Kingdom: Prevalence of antibody to HIV in haemophilics in the United Kingdom, a second survey. Clin Lab Haematol 10:187-191, 1988.

9. Schramm W, M Roggendorf, F Rommel, R Kammerer, H Pohlman, R Rasshofer, L Gurtler, F Deinhardt: Prevalence of antibodies to hepatitis C virus (HCV) in haemophiliacs. Blut 59:390-392, 1989.

10. Rumi MG, M Colombo, A Gringeri, PM Mannucci: High prevalence of antibody to hepatitis $\mathrm{C}$ virus in multitransfused hemophiliacs with normal transaminase levels. Ann Intern Med 112:379$380,1990$.

11. Triger DR: Chronic liver disease in haemophiliacs. Br J Haematol 74:241-245, 1990

12. Kasper CK, LM Aledort, RB Counts, JR Edson, J Fratantoni, D Green, JW Hampton, MW Hilgartner, J Lazerson, PH Levine, CW McMillan, JG Pool, SS Shapiro, NR Shulman, J van Eys: A more uniform measurement of factor VIII inhibitors. Thromb Diath Haemorrh 34:875-876, 1975.

13. Kasper CK, JB Graham, PBA Kernoff, MJ Larrieu, KA Rickard, PM Mannucci: Hemophilia: State of the art of hematologic care. Vox Sang 56:141-144, 1989
14. Aronstam A, M Wassef, Z Hamad, J Cartlidge, D McLellan: A double-blind controlled trial of two dose levels of factor VIII in the treatment of high risk haemarthroses in haemophilia A. Clin Lab Haematol 5:157-163, 1983.

15. Cohen H, PBA Kernoff: Plasma, plasma products and indications for their use. Br Med J 300:803-806, 1990.

16. Mannucci PM, M Colombo: Virucidal methods for clotting factor concentrates. Lancet 2:782-785, 1988.

17. Brettler DB, PH Levine: Factor concentrates for treatment of hemophilia: Which one to choose? Blood 73:2067-2073, 1989.

18. Roberts HR: The treatment of hemophilia: Past tragedy and future promise. N Engl J Med 321:1188-1190, 1989.

19. Mannucci PM: Desmopressin: A nontransfusional form of treatment for congenital and acquired bleeding disorders. Blood 72:1449-1455, 1988.

20. Kasper CK: The therapy of factor VIII inhibitors. In: Zimmerman TS, ZM Ruggeri (Eds): Coagulation and Bleeding Disorders: The Role of Factor VIII and von Willebrand Factor. Marcel Dekker, New York 1989, pp 59-75.

21. Anson DS, RA Hock, D Austen, KJ Smith, GG Brownlee, IM Verma, AD Miller: Towards gene therapy for hemophilia B. Mol Biol Med 4:11-20, 1987.

22. Hoeben RC, N van Tilburg, E Briet, H Van Ormondt, AJ Van der $\mathrm{Eb}$ : Towards gene therapy in hemophilia A: Expression of factor VIII with a retroviral vector system. Thromb Haemost 62:209 (Abst. 636), 1989.

23. Report of the WHO Expert Committee on Disability Prevention and Rehabilitation. Technical Report Series 668, Geneva, 1981.

24. Sethi PK: Technological choices in prosthetics and orthotics for developing countries. Prosthet Orthot Int 13:117-124, 1989.

25. Methods for the detection of haemophilia carriers. Bull WHO 55:675-702, 1977.

26. Graham JB, CR Rizza, J Chediak, PM Mannucci, E Briet, R Ljung, CK Kasper, EM Essien, PP Green: Carrier detection in hemophilia A: A cooperative international study. Blood 67:1554 1559, 1986.

27. Mibashan RS, IR Peake, CH Rodeck, JK Thumpston, RA Furlong, R Gorer, L Bains, AL Bloom: Dual diagnosis of prenatal haemophilia by measurement of fetal factor VIIIC and VIIIC antigen (VIIIAg). Lancet 2:994-997, 1980.

28. Tuddenham EGD: Factor VIII and haemophilia A. Balliere's Clin' Haematol 2:849-877, 1989.

29. Gianelli F: Factor IX. Balliere's Clin Haematol 2:821-848, 1989.

30. Mariani G, A Chistolini, HJ Hassan, E Gallo, G Xigen, M Papacchini, T Di Paolantonio, A Fantoni: Carrier detection for hemophilia B: Evaluation of multiple polymorphic sites. Am J Hematol 33:1-7, 1990.

31. White GC II, CB Shoemaker: Factor VIII gene and hemophilia A. Blood 73:1-12, 1989.

32. Gitschier J, S Kogan, B Levinson, EGD Tuddenham: Mutations of factor VIII cleavage sites in hemophilia A. Blood 72:1022-1028, 1988.

33. Higuchi M, L Kochhan, R Schwaab, H Egli, HH Brackmann, J Horst, K Olek: Molecular defects in hemophilia A: Identification and characterization of mutations in the factor VIII gene and family analysis. Blood 74:1045-1051, 1989.

34. Koeberl DD, CDK Bottema, J-M Buerstedde, SS Sommer: Functionally important regions of the factor IX gene have a low rate of polymorphism and a high rate of mutation in the dinuclotide $\mathrm{CpG}$. Am J Hum Genet 45:448-457, 1989.

35. Reiss J, U Neufeldt, K Wieland, B Zoll: Diagnosis of haemophilia $\mathrm{B}$ using the polymerase chain reaction. Blut 60:31-16, 1990.

36. Rickard KA: IHTC: Celebrating two decades of committment in haemophilia care. Life Paths (World Federation of Haemophilia) 2:1, 1990 . 